\title{
HIV Diagnoses, Prevalence and Outcomes in Nine Southern States
}

\author{
Susan Reif • Brian Wells Pence • Irene Hall • \\ Xiaohong Hu $\cdot$ Kathryn Whetten $\cdot$ Elena Wilson
}

Published online: 19 December 2014

(c) The Author(s) 2014. This article is published with open access at Springerlink.com

\begin{abstract}
A group of nine states in the Southern United States, hereafter referred to as the targeted states, has experienced particularly high HIV diagnosis and case fatality rates. To provide additional information about the HIV burden in this region, we used CDC HIV surveillance data to examine characteristics of individuals diagnosed with HIV in the targeted states (2011), 5-year HIV and AIDS survival, and deaths among persons living with HIV (2010). We used multivariable analyses to explore the influence of residing in the targeted states at diagnosis on deaths among persons living with HIV after adjustment for demographics and transmission risk. In 2011, the targeted states had a higher HIV diagnosis rate $(24.5 / 100,000$ population) than the US overall $(18.0 / 100,000)$ and higher proportions than other regions of individuals diagnosed with HIV who were black, female, younger, and living in suburban and rural areas. Furthermore, the targeted states had lower HIV and AIDS survival proportions (0.85, 0.73, respectively) than the US overall $(0.86,0.77$, respectively) and the highest death rate among persons living with HIV of any US region. Regional differences in demographics
\end{abstract}

S. Reif $(\square) \cdot$ K. Whetten $\cdot$ E. Wilson

Center for Health Policy and Inequalities Research,

Duke University, 310 Trent Drive, Durham, NC 27708, USA

e-mail: susan.reif@duke.edu

\section{B. W. Pence}

Department of Epidemiology, University of North Carolina School of Public Health, 135 Dauer Drive, Chapel Hill, NC 27599-2089, USA

I. Hall $\cdot$ X. Hu

HIV Incidence and Case Surveillance Branch, Division of HIV/ AIDS Prevention, National Center for HIV/AIDS, Viral Hepatitis, STD, and TB Prevention, 1600 Clifton Road, MS E 47, Atlanta, GA 30333, USA and transmission risk did not explain the higher death rate among persons living with HIV in the targeted states indicating that other factors contribute to this disparity. Differences in characteristics and outcomes of individuals with HIV in the targeted states are critical to consider when creating strategies to address HIV in the region, as are other factors identified in previous research to be prominent in the region including poverty and stigma.

Keywords HIV · AIDS - Southern United States · Mortality $\cdot$ HIV diagnosis

\section{Background}

The Southern United States (US) has been consistently identified as being disproportionately affected by HIV. In 2010, persons living in the South had the highest HIV and AIDS diagnosis rates and the highest number of individuals living with HIV of all US regions [1,2]. Although only $37 \%$ of the US population resides in the South, nearly half (49\%) of individuals living with HIV in 2010 were diagnosed in the South [3]. Persons living with HIV who were diagnosed in the South also had the lowest 3-year HIV survival rates (2002-2006) according to a Centers for Disease Control (CDC)-authored publication [2]. The authors of this publication argued that focus on this area of high HIV burden is warranted to successfully address HIV disease.

A group of Southern states are disproportionally affected by HIV and share certain characteristics, such as overall poor health of the population, high poverty rates, and negative health outcomes for those infected with HIV [4]. For the purpose of this article, these states are referred to as the "targeted states" and include Alabama, Florida, 
Georgia, Louisiana, Mississippi, North Carolina, South Carolina, Tennessee and Texas. These states have also been referred to as belonging to the "Deep South" [5]. The Deep South has been defined as a region whose states have a shared history of active promotion of slavery and a strong agricultural and economic base in cotton and tobacco [6]. The targeted states have higher levels of STDs and individuals without health insurance than other US regions, including the rest of the Southern states $[4,7-10]$ as well as high levels of HIV-related stigma [11]. These factors are implicated in negatively influencing HIV transmission and outcomes [4, 12-16].

When grouped as a region, the targeted states have the highest HIV diagnosis rates of any US region, including the rest of the Southern states (2008-2011) [7, 10]. In addition, previous research has found HIV case fatality rates, which are the number of deaths due to HIV among individuals living with HIV in a given year, to be high in the targeted states region, as eight of ten states with the highest HIV case fatality rates from 2002 to 2006 were targeted states [17].

These HIV epidemiologic data from the targeted states suggest a need for increased efforts to address HIV in this region. To create strategies for effective intervention, more detailed information regarding the characteristics and health outcomes of HIV-infected individuals in the targeted states region is needed. This manuscript addresses this information gap, reporting on HIV surveillance data to examine the demographic and HIV risk characteristics of individuals diagnosed and living with HIV in the targeted states region and to compare these to characteristics of persons diagnosed and living with HIV in other US regions. In addition, we examined HIV and AIDS survival and deaths among persons living with HIV in the targeted states region and compared these rates to those in other US regions. Finally, we examined the influence of residing in the targeted states region at HIV diagnosis on deaths among persons living with HIV after adjustment for individual characteristics including demographics and transmission risk category.

\section{Methods}

Data from the Center for Disease Control and Prevention's (CDC) National HIV Surveillance System were used to examine rates of HIV diagnosis, prevalence and survival among persons diagnosed with HIV [1]. All US states and the District of Columbia report information on persons diagnosed with HIV infection to the CDC in a uniform format and without identifying information.

We analyzed data on adults and adolescents (aged 13 years and older) diagnosed with HIV infection regardless of stage of disease at diagnosis through December 2011, and reported to the CDC through June 2012. We determined the number and rate of persons living with a diagnosis of HIV infection at the end of December 2010 to allow sufficient time for reporting of deaths. Stage of disease at diagnosis was classified according to the reported CD4 T-lymphocyte count or percentage, or documentation of an AIDS-defining condition at or within 3 months of HIV diagnosis.

Persons were assigned to states based on residence at diagnosis. The US regions were defined using the US Census definition. These include Northeast (Connecticut, Maine, Massachusetts, New Hampshire, New Jersey, New York, Pennsylvania, Vermont, and Rhode Island); Midwest (Illinois, Indiana, Iowa, Kansas, Michigan, Minnesota, Missouri, Nebraska, North Dakota, Ohio, South Dakota, and Wisconsin); South (Alabama, Arkansas, Delaware, Florida, Georgia, Kentucky, Louisiana, Maryland, Mississippi, North Carolina, Oklahoma, South Carolina, Tennessee, Texas, Virginia, and West Virginia); and West (Alaska, Arizona, California, Colorado, Hawaii, Idaho, Montana, Nevada, New Mexico, Oregon, Utah, Washington, and Wyoming). Targeted states include Alabama, Florida, Georgia, Louisiana, Mississippi, North Carolina, South Carolina, Tennessee and Texas.

Transmission categories were based on CDC's hierarchical classification system. [male-to-male sexual contact (men who have sex with men, MSM); injection drug use; MSM who also inject drugs; heterosexual contact with a person known to have or be at high risk for HIV infection; and other] [1]. We used the standard definition of Metropolitan Statistical Area (MSA) category at diagnosis as either urban (metropolitan area $>500,000$ population), suburban (metropolitan area of 50,000-499,999 population) or rural (nonmetropolitan population) [18].

HIV diagnosis and prevalence rates per 100,000 population were calculated using official estimates from the US Census Bureau [19]. In addition, HIV diagnosis and prevalence rates in the targeted states were stratified by race/ethnicity, age, sex, and MSA category (rural, suburban, and urban). Population denominators were not available from the US Census or other data sources to determine rates by transmission category so only proportions are included [1]. Analyses were adjusted for delays in reporting diagnoses and deaths and for missing risk factor information, but not for incomplete reporting [1].

We determined 5-year survival probabilities among persons diagnosed with HIV during 2003-2004 with Kaplan-Meier survival analyses [20]. A similar methodology was used to examine 5-year survival after an AIDS diagnosis in 2003-2004.

Death rates among persons living with HIV for 2010 were calculated as the number of deaths per 1,000 persons living with HIV. Rate ratios and confidence intervals were 
calculated to examine differences in deaths among persons living with HIV by sex, race/ethnicity, MSA category, transmission category and geographic region at diagnosis. In addition, adjusted death rates among persons living with HIV and rate ratios were estimated to examine geographical differences in deaths among persons living with HIV after adjustment for regional differences in the distribution of sex, race/ethnicity, transmission category, age, and MSA category.

\section{Results}

HIV Diagnoses (2011) in the Targeted States (Table 1)

In $2011,17,732$ persons were diagnosed with HIV in the targeted states, representing $38 \%$ of those diagnosed with HIV in the United States (Table 1). Nearly one-quarter (23\%) were female and a majority, $57 \%$, were black/ African American (hereafter referred to as black) The highest percentage of new diagnoses in the targeted states region, $27 \%$, was among persons aged 25-34 years; however, nearly one-quarter, $23 \%$, were aged $13-24$ years. The percentages of HIV diagnoses occurring among women, blacks, and individuals aged 13-24 were higher in the targeted states than the US average (Table 1).

The HIV diagnosis rate among blacks in the targeted states $(72.9$ per 100,000$)$ was higher than the overall US rate (70.0) but somewhat lower than the rest of the South (76.5) and the Northeast (81.8). The diagnosis rate among Hispanics or Latinos in the targeted states region (24.5) was comparable to the US rate (24.9). For individuals aged 13-24, the targeted states had a higher HIV diagnosis rate $(28.2 / 100,000)$ than the overall US rate $(19.2 / 100,000)$ and the targeted state HIV diagnosis rate among this age group was more than double the rate in the West and Midwest. The HIV diagnosis rate was also the highest in the targeted states region for all other age ranges when compared to other regions.

A lower percentage of persons diagnosed with HIV in 2011 in the targeted states resided in urban areas (72\%) compared with the United States overall (82\%). In the targeted states region, $17 \%$ of persons diagnosed with HIV resided in suburban areas and $11 \%$ in rural areas compared to 11 and $7 \%$ of persons diagnosed in the entire United States, respectively. In the rest of the South, only $8 \%$ of persons diagnosed with HIV lived in suburban areas and $7 \%$ in rural areas. Although a lower percentage of HIV diagnoses in the targeted Southern states were among individuals living in urban areas compared to the United States overall, the targeted states had a higher HIV diagnosis rate (29.6/ $100,000)$ in urban areas when compared to the United States overall and the Northeast region (22.5 and 23.4 respectively).
The targeted states region also had a higher HIV diagnosis rate in suburban areas $(18.6 / 100,000)$ and rural areas (14.4/ $100,000)$ compared to the United States overall (11.1 and 7.3 respectively).

Among both men and women, the targeted states had a higher percentage of diagnoses that were attributed to heterosexual contact (14.5 and $88.3 \%$ respectively) and a lower percentage attributed to injection drug use (IDU) (4.3 and $11.5 \%$ respectively) when compared to the United States overall for heterosexual contact (11.7 and $85.7 \%$ respectively) and IDU (5.6 and $14.1 \%$ respectively).

\section{HIV Prevalence, Year End 2010 (Table 2)}

In 2010 , there were an estimated 285,677 persons living with HIV in the targeted states; the highest number of any US region. As with HIV diagnosis, the targeted states had a higher percentage of individuals living with HIV that were female $(27.7 \%)$ than the overall United States $(24.9 \%)$ (Table 2). The targeted states also had a higher percentage of individuals living with HIV who were black $(54.2 \%)$ than the United States overall $(43.6 \%)$ but less than the rest of the South $(63.2 \%)$. In addition, the targeted states had a lower percentage of males and females living with HIV with a transmission category of IDU and a greater percentage with heterosexual contact as the reported transmission category than the United States overall and the rest of the Southern states. Over one-quarter of individuals living with HIV in the targeted states were living in suburban or rural areas (15\% suburban and $11 \%$ rural) at the time of diagnosis, which was the largest percentage of individuals living with HIV that had been diagnosed outside of urban areas in any region.

The HIV prevalence rates for both men $(595.9 / 100,000)$ and women $(215.4 / 100,000)$ were higher in the targeted states than the US average among men $(521.0 / 100,000)$ and women $(164.4 / 100,000)$ and all other regions except for the Northeast (717.6 for men and 291.7 for women). For the age ranges $35-44,45-54$, and over 55, the targeted states had prevalence rates higher than the US average but substantially lower than the Northeast. However, the HIV prevalence rate among 25-34 year olds was higher in the targeted states $(407.5$ per 100,000$)$ than the Northeast $(359.9$ per 100,000$)$ and the prevalence rate among 13-24 year olds was comparable between the targeted states (101.3) and Northeast (106.5). Finally the HIV prevalence rate in rural and suburban areas was higher in the targeted states in comparison to the Northeast and all other regions.

\section{Survival Among Persons Diagnosed with HIV}

The 5-year survival in the targeted states was equal for men and women and decreased with age (Table 3). Blacks had 
Table 1 Characteristics of individuals diagnosed with HIV in selected states in the Southern United States ${ }^{\mathrm{a}}$ and US regions, 2011

\begin{tabular}{|c|c|c|c|c|c|c|c|c|c|c|c|c|c|c|}
\hline & \multicolumn{2}{|c|}{$\begin{array}{l}\text { Targeted } \\
\text { States }\end{array}$} & \multicolumn{2}{|c|}{$\begin{array}{l}\text { United } \\
\text { States }\end{array}$} & \multicolumn{2}{|l|}{ South } & \multicolumn{2}{|c|}{$\begin{array}{l}\text { South } \\
\text { non-targeted } \\
\text { states }\end{array}$} & \multicolumn{2}{|c|}{ Northeast } & \multicolumn{2}{|c|}{ Midwest } & \multicolumn{2}{|l|}{ West } \\
\hline & $\%$ & Rate & $\%$ & Rate & $\%$ & Rate & $\%$ & Rate & $\%$ & Rate & $\%$ & Rate & $\%$ & Rate \\
\hline \multicolumn{15}{|l|}{ Sex } \\
\hline Male & 76.7 & 38.7 & 79.1 & 29.2 & 76.5 & 36.9 & 75.7 & 31.5 & 75.9 & 31.9 & 80.8 & 17.9 & 88.3 & 25.5 \\
\hline Female & 23.3 & 11.1 & 20.9 & 7.4 & 23.5 & 10.7 & 24.3 & 9.6 & 24.1 & 9.4 & 19.2 & 4.1 & 11.7 & 3.3 \\
\hline \multicolumn{15}{|l|}{ Race/ethnicity } \\
\hline Black/African American & 56.5 & 72.9 & 46.6 & 70 & 58 & 73.7 & 63.6 & 76.5 & 43.9 & 81.8 & 48.6 & 53 & 18.3 & 57.8 \\
\hline Hispanic/Latino & 17.6 & 24.5 & 20.9 & 24.9 & 15.7 & 24.8 & 8.3 & 27.2 & 26 & 44.1 & 10.9 & 19 & 35.9 & 19.5 \\
\hline White & 23 & 9.5 & 28 & 7.7 & 23.3 & 8.8 & 24.3 & 7 & 25.6 & 7.4 & 35.6 & 4.8 & 37.4 & 9.7 \\
\hline Multiple races & 1.7 & 41.1 & 2 & 25.2 & 1.8 & 35.2 & 2.1 & 24.7 & 2.2 & 41.5 & 3 & 28 & 1.5 & 9.3 \\
\hline Other & 1.2 & 9.1 & 2.6 & 8.1 & 1.3 & 8.3 & 1.6 & 6.7 & 2.3 & 7.7 & 1.9 & 6.5 & 6.8 & 8.5 \\
\hline \multicolumn{15}{|l|}{ Age at diagnosis } \\
\hline $13-24$ & 23.3 & 28.2 & 21.4 & 19.2 & 23 & 26.9 & 22.2 & 22.8 & 18.8 & 19.6 & 25.4 & 13.7 & 17.2 & 11.9 \\
\hline $25-34$ & 27.1 & 40.9 & 27.9 & 31.1 & 26.9 & 38.9 & 26.2 & 32.7 & 27.1 & 35.9 & 28.2 & 19.5 & 31 & 25.7 \\
\hline $35-44$ & 21.3 & 32.6 & 22.4 & 25.7 & 21.2 & 31.3 & 21.1 & 27.3 & 23.3 & 30.5 & 21.6 & 15.5 & 24.8 & 22 \\
\hline $45-54$ & 19 & 27.4 & 19.2 & 20 & 19.3 & 26.5 & 20.5 & 23.7 & 20.3 & 22.8 & 17.5 & 10.8 & 18.8 & 16.1 \\
\hline $55+$ & 9.4 & 7.6 & 9.2 & 5.4 & 9.5 & 7.3 & 10 & 6.5 & 10.5 & 6.7 & 7.2 & 2.5 & 8.1 & 4 \\
\hline \multicolumn{15}{|l|}{ Transmission category } \\
\hline \multicolumn{15}{|l|}{ Male } \\
\hline Male-to-male sexual contact & 78.1 & NA & 79 & NA & 77.6 & NA & 75.8 & NA & 72.2 & NA & 83.9 & NA & 85.5 & NA \\
\hline Injection drug use & 4.3 & NA & 5.6 & NA & 4.6 & NA & 5.6 & NA & 9.8 & NA & 4.2 & NA & 4.6 & NA \\
\hline $\begin{array}{l}\text { Male-to-male sexual contact and } \\
\text { injection drug use }\end{array}$ & 3 & NA & 3.6 & NA & 3 & NA & 3.1 & NA & 2.9 & NA & 3.7 & NA & 5.7 & NA \\
\hline Heterosexual contact & 14.5 & NA & 11.7 & NA & 14.7 & NA & 15.3 & NA & 15 & NA & 7.9 & NA & 4.2 & NA \\
\hline Other $^{\mathrm{b}}$ & 0.1 & NA & 0.1 & NA & 0.1 & NA & 0.2 & NA & 0 & NA & 0.2 & NA & 0.1 & NA \\
\hline \multicolumn{15}{|l|}{ Female } \\
\hline Injection drug use & 11.5 & NA & 14.1 & NA & 11.6 & NA & 12.1 & NA & 16.9 & NA & 14.4 & NA & 20.5 & NA \\
\hline Heterosexual contact & 88.3 & NA & 85.7 & NA & 88.2 & NA & 87.8 & NA & 83.1 & NA & 85.3 & NA & 79 & NA \\
\hline Other $^{\mathrm{b}}$ & 0.1 & NA & 0.2 & NA & 0.1 & NA & 0.1 & NA & 0.1 & NA & 0.3 & NA & 0.5 & NA \\
\hline \multicolumn{15}{|l|}{ Residence at diagnosis } \\
\hline $\begin{array}{l}\text { Urban (MSAs with population } \\
\geq 500,000)\end{array}$ & 72.2 & 29.6 & 81.7 & 22.5 & 74.7 & 29.5 & 83.7 & 29.4 & 92.5 & 23.4 & 80.3 & 15.7 & 89.2 & 17.4 \\
\hline $\begin{array}{l}\text { Suburban (metropolitan areas with } \\
\text { population } 50,000-499,999 \text { ) }\end{array}$ & 16.5 & 18.6 & 11.1 & 11.1 & 14.7 & 16.5 & 7.9 & 8.8 & 4.8 & 9.4 & 12.5 & 6.2 & 7.9 & 6.8 \\
\hline Rural (nonmetropolitan areas) & 10.7 & 14.4 & 6.6 & 7.3 & 10 & 11.9 & 7.2 & 6.1 & 2.2 & 4.5 & 6.8 & 3.2 & 2.5 & 3.6 \\
\hline Unknown & 0.5 & - & 0.5 & - & 0.6 & - & 1.1 & - & 0.5 & - & 0.4 & - & 0.4 & - \\
\hline \multicolumn{15}{|l|}{ Stage of disease at diagnosis } \\
\hline Stage $3(\text { AIDS })^{\mathrm{c}}$ & 28.5 & NA & 28.7 & NA & 28.3 & NA & 27.8 & NA & 29.2 & NA & 28.4 & NA & 29.2 & NA \\
\hline Stage 1 or 2 or unknown & 71.5 & NA & 71.3 & NA & 71.7 & NA & 72.2 & NA & 70.8 & NA & 71.6 & NA & 70.8 & NA \\
\hline Total & 100 & 24.5 & 100 & 18 & 100 & 23.5 & 100 & 20.2 & 100 & 20.2 & 100 & 10.8 & 100 & 14.3 \\
\hline
\end{tabular}

lower survival proportions at 5 years $(0.84)$ than whites (0.87) in the targeted states. Reporting injection drug (IDU) use as transmission risk was associated with a substantially lower 5-year survival proportion (0.80) than heterosexual contact (0.88) among females. Among males, IDU as a transmission category was also associated with lower 5-year survival (0.80) than MSM (0.90) and heterosexual contact (0.83). Five-year survival among those diagnosed 
Table 2 HIV prevalence in selected Southern States ${ }^{\mathrm{a}}$ and other United States Regions, year-end 2010

\begin{tabular}{|c|c|c|c|c|c|c|c|c|c|c|c|c|c|c|}
\hline & \multicolumn{2}{|c|}{$\begin{array}{l}\text { Targeted } \\
\text { states }\end{array}$} & \multicolumn{2}{|c|}{ United States } & \multicolumn{2}{|l|}{ South } & \multicolumn{2}{|c|}{$\begin{array}{l}\text { Southern } \\
\text { non-target }\end{array}$} & \multicolumn{2}{|c|}{ Northeast } & \multicolumn{2}{|c|}{ Midwest } & \multicolumn{2}{|l|}{ West } \\
\hline & $\%$ & Rate & $\%$ & Rate & $\%$ & Rate & $\%$ & Rate & $\%$ & Rate & $\%$ & Rate & $\%$ & Rate \\
\hline \multicolumn{15}{|l|}{ Sex } \\
\hline Male & 72.3 & 595.9 & 75.1 & 521 & 72 & 575.7 & 71 & 514 & 69.5 & 717.6 & 78.9 & 294.8 & 87.4 & 493.2 \\
\hline Female & 27.7 & 215.4 & 24.9 & 164.4 & 28.0 & 211.1 & 29 & 198.2 & 30.5 & 291.7 & 21.1 & 75.0 & 12.6 & 70.0 \\
\hline \multicolumn{15}{|l|}{ Race/ethnicity } \\
\hline Black/African American & 54.2 & $1,144.9$ & 43.6 & $1,231.6$ & 56.2 & $1,184.9$ & 63.2 & $1,321.8$ & 42.9 & $1,966.7$ & 44.2 & 815.5 & 16.2 & $1,001.0$ \\
\hline Hispanic/Latino & 14.6 & 336.9 & 19.0 & 433.0 & 12.6 & 336.5 & 5.7 & 332.4 & 27.1 & $1,148.4$ & 9.5 & 288.9 & 27.7 & 297.0 \\
\hline White & 29.0 & 194.7 & 33.8 & 173.1 & 28.8 & 179.3 & 28.1 & 139.9 & 26.0 & 183 & 42.5 & 97.1 & 50.6 & 254.1 \\
\hline Multiple races & 1.5 & 622.1 & 1.9 & 474.2 & 1.6 & 536.6 & 1.9 & 386.5 & 2.9 & $1,334.5$ & 2.3 & 367.5 & 1.2 & 152.0 \\
\hline Other & 0.7 & 85.4 & 1.6 & 97.6 & 0.8 & 85.0 & 1.2 & 84.3 & 1.2 & 102.3 & 1.5 & 85.0 & 4.3 & 105.1 \\
\hline \multicolumn{15}{|l|}{ Age at end of year } \\
\hline $13-24$ & 5.2 & 101.3 & 4.5 & 74.4 & 5.1 & 97.8 & 4.9 & 86.8 & 4.2 & 106.5 & 5.4 & 48.6 & 2.9 & 38.1 \\
\hline $25-34$ & 16.5 & 407.5 & 14 & 295.1 & 16.1 & 385.9 & 14.6 & 319.8 & 10.9 & 359.9 & 15.6 & 183 & 12.8 & 208.2 \\
\hline $35-44$ & 28.1 & 692.0 & 26.7 & 565.1 & 27.8 & 665.9 & 26.4 & 584.8 & 24.3 & 765.7 & 28.1 & 332.4 & 26.8 & 458.2 \\
\hline $45-54$ & 33.6 & 780.5 & 35.5 & 685 & 34 & 759.6 & 35.3 & 697.9 & 37.5 & $1,026.9$ & 34.2 & 348.5 & 36.9 & 607.1 \\
\hline $55+$ & 16.6 & 222.3 & 19.3 & 216.7 & 17.1 & 220.8 & 18.7 & 216.2 & 23.0 & 363.1 & 16.7 & 98.5 & 20.6 & 202.7 \\
\hline \multicolumn{15}{|l|}{ Transmission category } \\
\hline \multicolumn{15}{|l|}{ Male } \\
\hline $\begin{array}{l}\text { Male-to-male sexual } \\
\text { contact }\end{array}$ & 68.4 & NA & 67.7 & NA & 67.2 & NA & 63.0 & NA & 55.1 & NA & 75.2 & NA & 78.4 & NA \\
\hline Injection drug use & 9.3 & NA & 13.0 & NA & 10.6 & NA & 15.5 & NA & 25.0 & NA & 8.6 & NA & 6.4 & NA \\
\hline $\begin{array}{l}\text { Male-to-male sexual } \\
\text { contact and injection } \\
\text { drug use }\end{array}$ & 7.0 & NA & 7.5 & NA & 7.0 & NA & 6.9 & NA & 5.7 & NA & 7.7 & NA & 10.5 & NA \\
\hline Heterosexual contact & 14.5 & NA & 10.8 & NA & 14.3 & NA & 13.6 & NA & 12.6 & NA & 7.4 & NA & 4.0 & NA \\
\hline Other $^{\mathrm{b}}$ & 0.9 & NA & 1.1 & NA & 0.9 & NA & 1.1 & NA & 1.6 & NA & 1.1 & NA & 0.6 & NA \\
\hline \multicolumn{15}{|l|}{ Female } \\
\hline Injection drug use & 18.0 & NA & 24.9 & NA & 19.7 & NA & 25.5 & NA & 32.6 & NA & 21.9 & NA & 26.8 & NA \\
\hline Heterosexual contact & 79.8 & NA & 72.3 & NA & 78 & NA & 72.2 & NA & 63.9 & NA & 75.5 & NA & 69.6 & NA \\
\hline Other $^{\mathrm{b}}$ & 2.2 & NA & 2.8 & NA & 2.2 & NA & 2.2 & NA & 3.5 & NA & 2.6 & NA & 3.6 & NA \\
\hline \multicolumn{15}{|l|}{ Residence at diagnosis } \\
\hline $\begin{array}{l}\text { Urban (MSAs with } \\
\quad \text { population } \geq 500,000)\end{array}$ & 73.6 & 493.9 & 82.8 & 428.8 & 75.6 & 496.7 & 82.6 & 505.7 & 91.4 & 568.1 & 79.1 & 261.1 & 89.2 & 340.0 \\
\hline $\begin{array}{l}\text { Suburban (metropolitan } \\
\text { areas with population } \\
50,000-499,999)\end{array}$ & 14.9 & 271.3 & 10.0 & 185.6 & 13.5 & 248.2 & 8.6 & 164.4 & 4.9 & 236.7 & 13.0 & 109.2 & 7.5 & 126.9 \\
\hline $\begin{array}{l}\text { Rural (nonmetropolitan } \\
\text { areas) }\end{array}$ & 10.6 & 231.6 & 6.2 & 127.7 & 9.8 & 192.2 & 6.9 & 101.0 & 2.7 & 137.7 & 6.8 & 53.5 & 2.7 & 75.9 \\
\hline Unknown & 0.9 & - & 1.0 & - & 1.1 & - & 1.8 & - & 1.0 & - & 1.1 & - & 0.6 & - \\
\hline Total & 100 & 400.3 & 100 & 338.3 & 100 & 388.2 & 100 & 351.5 & 100 & 496.6 & 100 & 182.3 & 100 & 279.8 \\
\hline
\end{tabular}

${ }^{a}$ Includes Alabama, Florida, Georgia, Louisiana, Mississippi, North Carolina, South Carolina, Tennessee, Texas

${ }^{\mathrm{b}}$ Includes hemophilia, blood transfusion, perinatal exposure, and risk factor not reported or not identified

in rural areas of the targeted states (0.82) was lower than among those diagnosed in urban areas of the targeted states (0.86).

In the targeted states, the 5-year survival proportion after HIV diagnosis (for diagnoses in 2003-2004) was lower $(0.85)$ compared with the non-targeted states $(0.87)$.
There were differences in survival proportions between the targeted states. Texas had the highest 5-year HIV survival proportion (0.87), which was the same as the survival proportion for the non-targeted states. Louisiana had the lowest 5 -year survival proportion $(0.81)$ followed by Mississippi (0.83) and South Carolina (0.84). 
Table 3 Survival for more than 60 months after a diagnosis of AIDS or a diagnosis of HIV infection, adults and adolescents (aged 13 years or over) diagnosed in 2003-2004 in selected Southern States ${ }^{\mathrm{a}}$ and other United States Regions

\begin{tabular}{|c|c|c|}
\hline & \multicolumn{2}{|c|}{ Proportion survived } \\
\hline & $\begin{array}{l}\text { AIDS } \\
\text { survival }\end{array}$ & $\begin{array}{l}\text { HIV } \\
\text { survival }\end{array}$ \\
\hline United States $^{\mathrm{b}}$ & 0.77 & 0.86 \\
\hline Targeted states ${ }^{\mathrm{b}}$ & 0.73 & 0.85 \\
\hline \multicolumn{3}{|l|}{ State of residence } \\
\hline Alabama & 0.69 & 0.86 \\
\hline Florida & 0.72 & 0.85 \\
\hline Georgia & 0.75 & 0.86 \\
\hline Louisiana & 0.67 & 0.81 \\
\hline Mississippi & 0.68 & 0.83 \\
\hline North Carolina & 0.74 & 0.85 \\
\hline South Carolina & 0.73 & 0.84 \\
\hline Tennessee & 0.72 & 0.85 \\
\hline Texas & 0.76 & 0.87 \\
\hline \multicolumn{3}{|l|}{ Targeted states ${ }^{b}$} \\
\hline \multicolumn{3}{|l|}{ Sex } \\
\hline Male & 0.74 & 0.85 \\
\hline Female & 0.71 & 0.85 \\
\hline \multicolumn{3}{|l|}{ Race/ethnicity } \\
\hline Black/African American & 0.70 & 0.84 \\
\hline Hispanic/Latino & 0.78 & 0.87 \\
\hline White & 0.76 & 0.87 \\
\hline Multiple races & 0.73 & 0.84 \\
\hline Other & 0.78 & 0.89 \\
\hline \multicolumn{3}{|l|}{ Age at diagnosis } \\
\hline $13-24$ & 0.83 & 0.95 \\
\hline $25-34$ & 0.79 & 0.91 \\
\hline $35-44$ & 0.75 & 0.86 \\
\hline $45-54$ & 0.67 & 0.78 \\
\hline $55+$ & 0.52 & 0.61 \\
\hline \multicolumn{3}{|l|}{ Transmission category } \\
\hline \multicolumn{3}{|l|}{ Male adult or adolescent } \\
\hline Male-to-male sexual contact & 0.79 & 0.90 \\
\hline Injection drug use & 0.66 & 0.80 \\
\hline $\begin{array}{l}\text { Male-to-male sexual contact and } \\
\text { injection drug use }\end{array}$ & 0.75 & 0.88 \\
\hline Heterosexual contact & 0.72 & 0.83 \\
\hline Other $^{\mathrm{c}}$ & 0.64 & 0.76 \\
\hline \multicolumn{3}{|l|}{ Female adult or adolescent } \\
\hline Injection drug use & 0.63 & 0.80 \\
\hline Heterosexual contact & 0.75 & 0.88 \\
\hline Other $^{c}$ & 0.66 & 0.82 \\
\hline \multicolumn{3}{|l|}{ Residence at diagnosis } \\
\hline $\begin{array}{l}\text { Urban (MSAs with population } \\
\geq 500,000 \text { ) }\end{array}$ & 0.73 & 0.86 \\
\hline $\begin{array}{l}\text { Suburban (metropolitan areas with } \\
\text { population 50,000-499,999) }\end{array}$ & 0.71 & 0.84 \\
\hline
\end{tabular}

Table 3 continued

\begin{tabular}{|c|c|c|}
\hline & \multicolumn{2}{|c|}{ Proportion survived } \\
\hline & $\begin{array}{l}\text { AIDS } \\
\text { survival }\end{array}$ & $\begin{array}{l}\text { HIV } \\
\text { survival }\end{array}$ \\
\hline Rural (nonmetropolitan areas) & 0.71 & 0.82 \\
\hline Unknown & 0.82 & 0.93 \\
\hline
\end{tabular}

a Includes Alabama, Florida, Georgia, Louisiana, Mississippi, North Carolina, South Carolina, Tennessee, Texas

b Excludes cases diagnosed prior to the earlier date of HIV codebased and name-based reporting dates. Therefore, (1) 'United States' excludes PA, (2) 'Targeted states' includes all the nine targeted-states (GA has only 2004 data), and (3) 'Other states' includes the other states (40 states + DC excluding PA) of (1)

c Includes hemophilia, blood transfusion, perinatal exposure, and risk factor not reported or not identified

\section{Survival Among Persons Ever Diagnosed with AIDS}

In the targeted states, females had a lower 5-year AIDS survival proportion (0.71) than males (0.74) (Table 3). Blacks had the lowest survival proportion $(0.70)$ of any race/ethnicity and survival decreased with age. For example, in the targeted states among those diagnosed with AIDS who were 55 or older, $48 \%$ died within 5 years of AIDS diagnosis whereas $17 \%$ of those 18-24 died within 5 years of AIDS diagnosis. The 5-year survival proportion after an AIDS diagnosis was lower in suburban and rural areas $(0.71)$ than in urban areas $(0.73)$.

For 5-year survival after an AIDS diagnosis, the targeted states had the lowest survival proportion of any region (0.73), indicating that $27 \%$ of those diagnosed with AIDS in 2003-2004 had died within 5 years. The 5-year AIDS survival proportion was higher for the entire United States (0.77), the Northeast (0.79), the Midwest (0.78) and the West (0.82). Similar to HIV survival, there was variation in 5-year AIDS survival in the targeted states with Louisiana having the lowest survival rate at 0.67 , indicating that onethird of individuals diagnosed with AIDS in Louisiana in 2003-2004 had died within 5 years of diagnosis. The next lowest survival proportions in the targeted states were Mississippi (0.68) and Alabama (0.69). No targeted state had a 5-year AIDS survival proportion at or above the overall US survival proportion.

\section{Deaths Among Persons Living with HIV}

The death rate among persons living with HIV was higher in the targeted states (27.3 per 1,000 persons estimated to be living with HIV) than in any other region (non-targeted Southern states: 24.6; Northeast 24.7, Midwest 20.7, West 18.8.) (Table 4). After adjustment for age, sex, 
Table 4 Death rate among persons living with HIV, adjusted and adjusted by United States region and by specific characteristics within selected Southern States ${ }^{\mathrm{a}}, 2010$

\begin{tabular}{|c|c|c|c|}
\hline & $\begin{array}{l}\text { Unadjusted rate } \\
\text { Rate of deaths } 2010 \text { (among 1,000 PLWH } \\
\text { and new diagnoses during 2010) }\end{array}$ & $\begin{array}{l}\text { Adjusted rate }^{\mathrm{d}} \\
\text { Rate of deaths } 2010 \text { (among 1,000 PLWH } \\
\text { and new diagnoses during 2010) }\end{array}$ & $\begin{array}{l}\text { Rate ratio } \\
\text { (CI) }\end{array}$ \\
\hline United States & 24.0 & & \\
\hline \multicolumn{4}{|l|}{ Region of residence } \\
\hline Northeast & 24.7 & 22.3 & $\begin{array}{l}0.77 \\
(0.74,0.80)\end{array}$ \\
\hline Midwest & 20.7 & 22.5 & $\begin{array}{l}0.77 \\
(0.74,0.81)\end{array}$ \\
\hline South & 26.7 & 28.0 & \\
\hline Targeted states ${ }^{\mathrm{a}}$ & 27.3 & 29.0 & Reference \\
\hline South non-targeted states & 24.6 & 24.0 & $\begin{array}{l}0.83 \\
\quad(0.79,0.87)\end{array}$ \\
\hline West & 18.8 & 21.2 & $\begin{array}{l}0.73 \\
(0.70,0.76)\end{array}$ \\
\hline \multicolumn{4}{|l|}{ State of residence } \\
\hline Alabama & 30.7 & & \\
\hline Florida & 29.1 & & \\
\hline Georgia & 28.4 & & \\
\hline Louisiana & 34.5 & & \\
\hline Mississippi & 28.6 & & \\
\hline North Carolina & 25.6 & & \\
\hline South Carolina & 29.6 & & \\
\hline Tennessee & 25.0 & & \\
\hline Texas & 22.2 & & \\
\hline \multicolumn{4}{|l|}{ Targeted states } \\
\hline \multicolumn{4}{|l|}{ Sex } \\
\hline Male & 26.9 & & \\
\hline Female & 28.2 & & \\
\hline \multicolumn{4}{|l|}{ Race/ethnicity } \\
\hline Black/African American & 30.0 & & \\
\hline Hispanic/Latino & 19.8 & & \\
\hline White & 25.5 & & \\
\hline Multiple races & 41.1 & & \\
\hline Other & 12.4 & & \\
\hline \multicolumn{4}{|l|}{ Age category } \\
\hline $13-24$ & 7.5 & & \\
\hline $25-34$ & 13.5 & & \\
\hline $35-44$ & 20.3 & & \\
\hline $45-54$ & 31.2 & & \\
\hline $55+$ & 56.9 & & \\
\hline \multicolumn{4}{|l|}{ Transmission category } \\
\hline \multicolumn{4}{|l|}{ Male adult or adolescent } \\
\hline Male-to-male sexual contact & 21.5 & & \\
\hline Injection drug use & 45.1 & & \\
\hline $\begin{array}{l}\text { Male-to-male sexual contact and } \\
\text { injection drug use }\end{array}$ & 36.1 & & \\
\hline Heterosexual contact & 36.0 & & \\
\hline Other $^{\mathrm{b}}$ & 26.3 & & \\
\hline Female adult or adolescent & & & \\
\hline
\end{tabular}


Table 4 continued

\begin{tabular}{|c|c|c|c|}
\hline & $\begin{array}{l}\text { Unadjusted rate } \\
\text { Rate of deaths } 2010 \text { (among 1,000 PLWH } \\
\text { and new diagnoses during 2010) }\end{array}$ & $\begin{array}{l}\text { Adjusted rate }^{\mathrm{d}} \\
\text { Rate of deaths } 2010 \text { (among 1,000 PLWH } \\
\text { and new diagnoses during 2010) }\end{array}$ & $\begin{array}{l}\text { Rate ratio } \\
(\mathrm{CI})\end{array}$ \\
\hline Injection drug use & 39.0 & & \\
\hline Heterosexual contact & 26.0 & & \\
\hline Other $^{\mathrm{b}}$ & 18.0 & & \\
\hline \multicolumn{4}{|l|}{ Residence at diagnosis } \\
\hline $\begin{array}{l}\text { Urban (MSAs with population } \\
\geq 500,000 \text { ) }\end{array}$ & 25.6 & & \\
\hline $\begin{array}{l}\text { Suburban (metropolitan areas with } \\
\text { population } 50,000-499,999 \text { ) }\end{array}$ & 32.5 & & \\
\hline Rural (nonmetropolitan areas) & 31.8 & & \\
\hline Unknown & 22.4 & & \\
\hline \multicolumn{4}{|c|}{ a Includes Alabama, Florida, Georgia, Louisiana, Mississippi, North Carolina, South Carolina, Tennessee, and Texas } \\
\hline \multicolumn{4}{|c|}{ b Includes hemophilia, blood transfusion, perinatal exposure, and risk factor not reported or not identified } \\
\hline \multicolumn{4}{|c|}{$\begin{array}{l}\text { c Rates are per } 1,000 \text { persons living with diagnosed HIV infection (PLWH), denominator was calculated as (No. of PLWH at the end } \\
2009+\text { new diagnoses during 2010) }\end{array}$} \\
\hline
\end{tabular}

transmission category, and area population size, these differences were accentuated or substantively unchanged and the death rate among persons living with HIV in the targeted states was significantly higher than in the other regions, e.g., adjusted rate ratio targeted states versus nontargeted Southern states [Rate Ratio 0.83; $95 \%$ confidence intervals (CI) 0.79, 0.87].

Within the targeted states, blacks had a higher death rate among persons living with HIV than whites (rate ratio 1.2, CI 1.12, 1.24; data not shown) and the death rate among persons living with HIV increased with age category. Persons with HIV infection attributed to injection drug use or heterosexual contact had higher death rates among persons living with HIV than persons with infection attributed to male-to-male sexual contact (1.97, CI 1.86, 2.10 and 1.36 , CI 1.29, 1.43 respectively). Suburban and rural residence at diagnosis significantly predicted greater death rates among persons living with HIV compared to urban residence at diagnosis (1.27, CI 1.121 .34 and 1.24, CI 1.16 1.33, respectively). Louisiana had the highest death rate among persons living with HIV and Texas had the lowest death rate among persons living with HIV of all targeted states.

\section{Discussion}

Our results indicate that in 2011 the targeted states had the highest HIV diagnosis rate of any US region. The percentage of individuals diagnosed with HIV in the targeted states was disproportionate to the population size, as $38 \%$ of individuals diagnosed with HIV resided in the targeted states, while the targeted states accounted for $28 \%$ of the US population [1, 3]. Persons diagnosed with HIV in the targeted states region reflected higher proportions of women, blacks, and individuals residing in suburban and rural areas than the overall United States.

In the targeted states, a higher proportion of persons diagnosed with HIV were adolescents and young adults than in the United States overall and HIV diagnosis rates among individuals 13-24 and 25-34 were higher than in other US regions. Higher HIV diagnosis rates among the younger age categories, particularly in the targeted states region and the rest of the South, could be attributed, in part, to lack of education about HIV transmission and less gravity placed on HIV infection due to improvements in available drug regimens [21]. The higher concentration of HIV in younger ages in the targeted states is an important factor to consider in prevention and treatment planning, as response to prevention education and interventions may differ by age [22]. Development and implementation of effective prevention and treatment strategies for the younger population will be critical to stemming HIV transmission in the targeted Southern states.

The targeted states had higher HIV diagnosis rates than all other regions in urban areas, as well in suburban and rural areas indicating that the targeted states region is grappling with significant and disproportionate HIV burden in both urban and more rural areas. Challenges to HIV prevention and care in rural and suburban areas of the targeted states, such as lack of transportation, lack of qualified providers, and HIV-related stigma [23-27], may 
provide some explanation for the study findings regarding lower 5-year HIV and AIDS survival proportions in the targeted states in comparison to other US regions. In addition, the death rate among persons living with HIV (2010) was found to be higher in the targeted states compared to the overall United States; with rural and suburban areas having higher death rates among persons living with HIV than urban areas of the targeted states. The higher death rate among persons living with HIV in the targeted states suggests a disconnect between diagnosis and maintenance of HIV care in this region, particularly in nonurban areas. Identifying effective ways to structure prevention and care services so that they address common barriers to care such as accessibility and pervasive stigma will be critical to improving HIV outcomes in rural and suburban areas of the targeted states [4, 11, 24, 26].

Regional differences in the characteristics of individuals living with HIV, including sex, race/ethnicity, mode of transmission, MSA category and age, did not explain the higher death rate among persons living with HIV in the targeted states compared with other US regions. Rather, the disparity in the death rate among persons living with HIV between the targeted states and other regions were substantively unchanged or accentuated after adjustment, suggesting that additional factors unmeasured in the data contribute to the greater risk of death among persons living with HIV in the targeted states. These contributing factors likely include characteristics of the targeted states such as lower levels of income, education, and insurance coverage and higher levels of HIV stigma and racism $[4,9,11,28,29]$. Previous research has consistently related HIV-related stigma to negative outcomes, including poor medication adherence and greater HIV risk behavior [12-16]. A recent qualitative study among young black MSM reported that HIV-related stigma and homophobia were related to sexual risk behavior, reluctance to obtain HIV testing or care, and poorer medication adherence [30]. An additional contributing factor may be the social class system unique to the US South that has traditionally allowed for little social mobility, along with marginalization of, and discrimination against certain groups and often resulting in distrust of systems of care among those in a lower social strata [31-33]. These societal factors have likely collectively contributed to creating an environment in the targeted states in which HIV infection is more likely and health outcomes for HIV-positive individuals are poorer than in other US regions. Additional research is needed to better determine and understand the factors that influence the higher death rate among persons living with HIV in the targeted states and to identify effective mechanisms to address known barriers including HIV stigma.

Among the targeted states, HIV survival and deaths among persons living with HIV were not uniform. For example, Texas had a 5-year AIDS survival proportion only slightly lower than the overall US survival proportion, while Louisiana had a substantially lower 5-year survival proportion. The study findings regarding deaths among persons living with HIV are consistent with an analysis by Hanna and Colleagues from an earlier period, 2001-2007, that found all targeted states but Texas to be in the ten states with the highest HIV case fatality rates in the United States [17]. The targeted states with the most concerning mortality statistics, particularly Louisiana, may especially be in need of focused attention on addressing the factors contributing to these concerning statistics.

The findings of this study must be considered in the context of the study limitations. The data were adjusted for delays in reporting, however, they were not adjusted for incomplete reporting, and may slightly underrepresent the actual number of HIV diagnoses in the time period of study. In addition, a portion of deaths may not be reported to the HIV surveillance system [1], affecting the estimate of the number of people living with HIV disease. Relocation is not always accounted for within the surveillance data. Furthermore, the data are presented according to the area of residence at diagnosis and may not reflect the current residence for persons living with a diagnosis of HIV infection. If these potential errors occur more frequently in specific geographical areas rather than occurring randomly throughout the United States they may affect the accuracy of regional comparisons. Finally, the HIV diagnosis data cannot accurately provide information on HIV incidence, as they reflect only diagnoses made rather than occurrence of new HIV infections.

In conclusion, HIV surveillance data indicate a disproportionate impact of HIV in the targeted Southern states in terms of higher HIV diagnosis and prevalence rates and greater HIV mortality. These findings signal a need for effective strategies to address HIV in this region. The characteristics of individuals diagnosed with HIV in the targeted state region differ from the overall United States, as a greater proportion of these individuals in the targeted states are black, female, and younger. The targeted states also have a higher proportion of those diagnosed and living with HIV in suburban and rural areas than any other US region. These differences are crucial to consider, as are other factors prominent in the targeted states including poverty and stigma [4, 34], when creating strategies to address HIV in this region.

Acknowledgments This research was commissioned by the AIDS Legal Project at Duke University through a Ford Foundation Grant (1115-1373). We wish to thank Donna Safley for her work in contributing to the study.

Open Access This article is distributed under the terms of the Creative Commons Attribution License which permits any use, 
distribution, and reproduction in any medium, provided the original author(s) and the source are credited.

\section{References}

1. Centers for Disease Control and Prevention. (2013). HIV Surveillance Report 2011, Vol 23. Retrieved from http://www.cdc. gov/hiv/topics/surveillance/resources/reports/. Accessed March 2013.

2. Prejean, J., Tang, T., \& Hall, I. (2012). HIV diagnoses and prevalence in the southern region of the United States, 2007-2010. Journal of Community Health, 38(3), 414-426.

3. Reif, S., Whetten, K., Wilson, E., et al. (2014). HIV/AIDS in Southern USA: A disproportionate epidemic. AIDS Care, 26(3), 351-359.

4. Reif, S., Whetten, K., Wilson, L., \& Gong, W. (2011). HIV/AIDS epidemic in the South reaches crisis proportions in the last decade. Durham. Retrieved from http://southernaidsstrategy.org/ research/. Accessed July 2012.

5. Merriam-Webster. (2011). Deep South. Encyclopedia Britannica. Retrieved from http://www.merriam-webster.com/dictionary/ deepsouth. Accessed November 2011.

6. Birdsall, S. \& Florin, J. (2011). The Deep South. Retrieved from http://countrystudies.us/united-states/geography-14.htm

7. NCHHSTP Atlas. (2014). Retrieved from http://www.cdc.gov/ nchhstp/atlas/

8. Kaiser Family Foundation. (2011). Statehealthfacts.org: Povery rates 2009-2010. Retrieved from http://www.statehealthfacts. org/comparetable.jsp?ind $=10 \&$ cat $=1 \&$ sub $=2 \& y r=252 \&$ typ $=2$. Accessed September 2012.

9. Kaiser Family Foundation. (2011). Uninsured estimates of the total population, American Community Survey (ACS), 2009. Retrieved from http://www.statehealthfacts.org/comparemaptable.jsp?ind= $882 \&$ cat $=3$. Accessed 2011 November.

10. Reif, S., Safley, D., Wilson, E., \& Whetten, K. (2014). HIV/AIDS in the Southern US: Trends from 2008-2011 show a consistent disproportionate epidemic. Retrieved from http://south ernaidsstrategy.org/research/

11. National Alliance of State and Territorial AIDS Directors. (2012). Stigma and the impact on public health. Ryan White HIV/AIDS Program Grantee Meeting, Washington, DC.

12. Wasserheit, J. (1992). Epidemiological synergy. Interrelationships between human immunodeficiency virus infection and other sexually transmitted diseases. Sexually Transmitted Diseases, 19(2), 61-77.

13. Whetten, K., Reif, S., Whetten, K., \& Murphy-McMillan, K. (2008). Trauma, mental health, distrust, and stigma among HIVpositive persons: Implications for effective care. Psychosomatic Medicine, 70, 531-538.

14. Vanable, P., Carey, M., Blair, D., \& Littlewood, R. (2008). Impact of HIV-related stigma on health behavior and psychological adjustment among HIV-positive men and women. AIDS and Behavior, 10(5), 473-482.

15. Rintamaki, L., Davis, T., Skripkauskas, S., Bennett, C., \& Wolf, M. (2006). Social stigma concerns and HIV medication adherence. AIDS Patient Care and STDs, 20(5), 359-368.

16. Mahajan, A., Sayles, J., Patel, V., et al. (2008). Stigma in the HIV/AIDS epidemic: A review of the literature and recommendations for the way forward. AIDS, 22(Suppl 2), S67-S79.
17. Hanna, D., Selik, R., Tang, T., \& Gange, S. (2011). Disparities among states in HIV-related mortality in persons with HIV infection, 37 US States, 2001-2007. AIDS, 26(1), 95-103.

18. Office of Management and Budget. (2009). Update of statistical area definitions and guidance on their uses. Retrieved from http:// www.whitehouse.gov/sites/default/files/omb/assets/bulletins/b1002.pdf

19. US Census Bureau. (2010). Population estimates: Dataset. Accessed 2014.

20. Bland, J., \& Altman, D. (1998). Survival probabilities (the Kaplan-Meier method). British Medical Journal, 317, 1575.

21. Rengel, M., Gavin, L., Reed, C., Fowler, M., \& Lee, L. (2006). Epidemiology of HIV and AIDS among adolescents and young adults in the United States. Journal of Adolescent Health, 39(2), 156-163.

22. Kingdon, M., Storholm, E., Halkitis, P., et al. (2013). Targeting HIV prevention messaging to a new generation of gay, bisexual and other young men who have sex with men. Journal of Health Communication, 18, 325-342.

23. Cohn, S., Klein, J., Van der Horst, C., \& Weber, D. (2001). The care of HIV-infected adults in rural areas of the United States. Journal of Acquired Immune Deficiency Syndromes, 28, 385-392.

24. Heckman, T., Somlai, A., Peters, J., et al. (1998). Barriers to care among persons living with HIV/AIDS in urban and rural areas. AIDS Care, 10, 365-375.

25. Golin, C., Isasi, F., Bontempi, J., \& Eng, E. (2002). Secret pills: HIV-positive patients' experiences taking antiretroviral medications in North Carolina. AIDS Education and Prevention, 14, 318-329.

26. Reif, S., Golin, C., \& Smith, S. (2005). Barriers to accessing HIV/ AIDS care in North Carolina: Rural and urban differences. AIDS Care, 17, 558-565.

27. Pellowski, J. (2013). Barriers to care for rural people living with HIV: A review of domestic research and health care models. Journal of the Association of Nurses in AIDS Care, 24(5), 422-437.

28. Kaiser Family Foundation. Median annual household income, 2007-2009. Retrieved from http://www.statehealthfacts.org/com paremaptable.jsp?ind $=15 \&$ cat $=1$. Accessed November 2011 .

29. US Census Bureau. (2012). Education attainment by State 1990 2009 (Table 233). Retrieved from, http://www.census.gov/compen dia/statab/2012/tables/12s0233.pdf. Accessed November 2014.

30. Arnold, E., Rebchook, G., \& Kegeles, S. (2014). Triply cursed: Racism, homophobia and HIV-related stigma are barriers to regular HIV testing, treatment adherence and disclosure among young Black gay men. Culture, Health and Sexuality, 16(6), 710-722.

31. MacKethan, L. (2004). Genres of southern literature. Southern space. Retrieved from http://southernspaces.org/2004/genressouthern-literature. Accessed November 2014.

32. Whetten, K., Leserman, J., Whetten, R., et al. (2006). Exploring lack of trust in care providers and the government as a barrier to health service use. American Journal of Public Health, 96(4), $716-721$.

33. Cobb, J. (1999). Redefining southern culture: Mind and identity in the modern South. Athens, GA: University of George Press.

34. Kaiser Family Foundation. (2011). Poverty rate by age, states (2009-2010), US (2010). Retrieved from http://www.state healthfacts.org/comparetable.jsp?ind $=10 \&$ cat $=1 \&$ sub $=2 \& \mathrm{yr}=$ 199\&typ=2. Accessed November 2011. 